\title{
Highly Efficient Grouping Strategy for the Analysis of Two-port Arbitrarily Shaped H-plane Waveguide Devices
}

\author{
Ángel Belenguer*, Héctor Esteban ${ }^{\dagger}$, Vicente E. Boria ${ }^{\dagger}$, Carmen Bachiller ${ }^{\dagger}$, and José V. Morro ${ }^{\dagger}$ \\ * Universidad de Castilla-La Mancha, Spain. angel.belenguer@uclm.es \\ † Universidad Politécnica de Valencia, Spain. hesteban@dcom.upv.es
}

\begin{abstract}
A new grouping strategy for the analysis of two-port arbitrarily shaped H-plane devices in rectangular waveguides is presented. The new grouping strategy accelerates a previous method based on the method of moments and the Green's function of an infinite line source placed inside two parallel plates. The computational cost of analyzing several H-plane filters of different geometries is reduced by about $50 \%$, while maximum accuracy is maintained, as a result of using the new grouping strategy.
\end{abstract}

Index Terms-Moment methods, Green function, rectangular waveguides, scattering, cavity resonator filters.

\section{INTRODUCTION}

$\mathbf{T}$ HE well known method of moments (MoM) [1] is typically employed for the analysis of antennas and scattering problems in free-space [2], [3] where no boundary conditions are imposed on the corresponding Green's function. However, the MoM can also be applied to the analysis of electromagnetic problems with boundary conditions, such as H-plane devices in waveguide technology [4]-[6], which are widely used in telecommunication applications, as can be inferred from several recent publications [7]-[11]. To apply the MoM to the analysis of rectangular waveguide H-plane devices, the Green's function of an infinite line source between two parallel plates must be used [12]. This Green's function, defined as the summation of a series of guided modes [13], has been previously used to solve the scattering of single [14], [15] or multiple [16], [17] H-plane posts in a rectangular waveguide. The convergence rate of the summation of this Green's function has been accelerated, either using the Kummer's transformation [14], [17], or by employing particular basis functions in the application of MoM [16]. Recently [18], the convergence rate of the MoM with the Green's function of a line source between parallel plates has been improved by first evaluating the integrals related to the application of MoM, and then summing the resulting terms, instead of first summing the Green's function and then solving the integral equations of MoM, as this was traditionally handled. This new approach simultaneously reduces the computational cost and increases accuracy, as the distributed sources can now be placed along the surface of the scatterer, and no singularity arises bacause it disappears through integration before the Green's function is summed. In this paper we significantly improve the numerical efficiency of the technique presented in [18] by grouping the radiation of neighbouring MoM contour cells.

The idea of grouping the electromagnetic fields produced by certain groups of cells is not new. This technique has been typically employed in the fast multipole method [19]-[22] and in the multilevel fast multipole algorithm [23]-[25], in both cases providing important efficiency improvements. These methods accelerate the solution of the matrix system provided by the MoM, by using an iterative technique such as conjugate gradients, biconjugate gradients, biconjugate gradients stabilized, and others [22], [26]-[29]. These iterative techniques do not directly solve the matrix system $\boldsymbol{Z} \boldsymbol{\alpha}=\boldsymbol{g}$ of MoM, but instead they approximate the solution $\alpha$ iteratively through multiple matrix-vector products of the form $\boldsymbol{Z} \boldsymbol{a}$. Again these products are not computed directly, but they are evaluated more efficiently thanks to a strategy based on three steps: aggregation of the radiation of neighbouring cells into a single radiation pattern; translation; and disaggregation of the incoming field into each MoM cell. Those grouping strategies imply a cost reduction of the matrix-vector product from $O\left(N^{2}\right)$ to $O\left(N^{3 / 2}\right)$ for the fast multipole method, and $O(N \log (N))$ for the multilevel fast multipole algorithm, where $N$ represents the number of discretization cells considered along the scatterer contours.

Due to this important cost reduction, the fast multipole method and the multilevel fast multipole algorithm are widely used in the analysis of complex and large scattering structures. Recent works are focused on improving the accuracy by refining several subprocesses of the grouping strategy, for example, by the anterpolation/interpolation of radiation diagrams of groups [30], [31], or by using better sets of basis functions in the MoM current expansion [32], [33]. Other works try to improve the efficiency by accelerating the convergence of the iterative algorithm used in the matrix system solution [34]-[36], or trying to parallelize the computations [37]-[41]. Finally, there are other papers that are focused on using these algorithms for analyzing new 2D or 3D structures in free space or in free semi-spaces, as in [42]-[46].

In this paper we propose a method for analyzing two-port arbitrarily shaped H-plane devices in rectangular waveguides. This new method uses the technique proposed in [18] for accelerating the convergence and improving the accuracy of the MoM using the Green's function of a line source among 
parallel plates waveguide. The numerical efficiency of this technique is improved here with the use of a new grouping strategy inspired by the fast multipole method.

\section{ACCELERATION PROCEDURE}

The arbitrarily shaped H-plane waveguide devices are analyzed by applying MoM (pulses as basis functions and deltas as test functions, that is, Point Matching) [1], which leads to the following matrix system,

$$
E_{i}=Z j
$$

Once this matrix system is solved, we obtain a set of coefficients $j$ which can be used to reconstruct the current induced over the scatterers contours due to the excitation $\boldsymbol{E}_{i}$. Using the coefficients $\boldsymbol{j}$ it is possible to compute the generalized scattering matrix of the global structure according to the algorithm recently presented in [18].

The most time consuming process of MoM is solving the matrix system. An alternative to directly solving the matrix system, especially with large matrix systems, is the use of an iterative algorithm such as conjugate gradients, biconjugate gradients, biconjugate gradients stabilized, and others [22], [26]-[29]. These algorithms approximate the solution $\boldsymbol{j}$ iteratively through multiple matrix-vector products of the form $Z \boldsymbol{a}$. These matrix products are also time consuming, but we can obtain an accurate approximation for them if we take into account that they represent the field scattered by the whole structure with current distribution $a$ at the center of each MoM cell. Using this physical interpretation for the product $\boldsymbol{Z} \boldsymbol{a}$ we propose a grouping strategy similar to that used in the fast multipole method. The main difference between those techniques and our method is that they are typically applied to open space problems (i.e. antennas) while our method will be applied to H-plane devices in a rectangular waveguide (see Fig. 1). As in the fast multipole method, we approximate the matrix product $\boldsymbol{Z} \boldsymbol{a}$ with a grouping strategy in three independent steps [20], [21]:

1) First, grouping of neighbour cells with the proper combination of their scattered fields into a single "modal radiation pattern".

2) Next, the "modal radiation pattern" is translated to the reference plane of each target group.

3) Finally, the summation of all the "modal radiation patterns" incident over each target group is evaluated at each one of the cells belonging to that group, and this completes a process which is equivalent to the matrixvector computation.

In the fast multipole method terminology, the first process is named "aggregation", the second one "translation", and the third and last one "disaggregation". We must adapt this grouping strategy to closed problems in order to achieve an important improvement in the global numerical efficiency.

\section{A. Aggregation}

In open space, where the field is expanded into open space modes that propagate radially, the grouping of the radiation of neighbour cells is based on a 2-D or 3-D proximity criterion.
However, inside an H-plane device in rectangular waveguide, the geometry is invariant in height ( $z$ dimension in Fig. 1). Considering that the exciting field is the fundamental $T E_{10}$ mode, which is also invariant along $z$, then all the scattered fields by the H-plane structure are also invariant in height ( $T E_{p 0}$ modes). Therefore the 3-D structure of Fig. 1(a) can be reduced to the equivalent 2-D problem of Fig. 1(b).

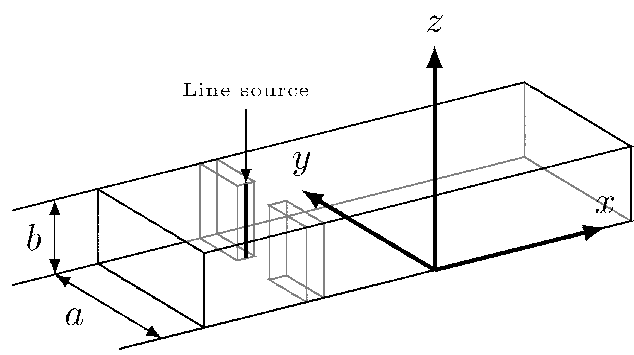

(a) 3-D view

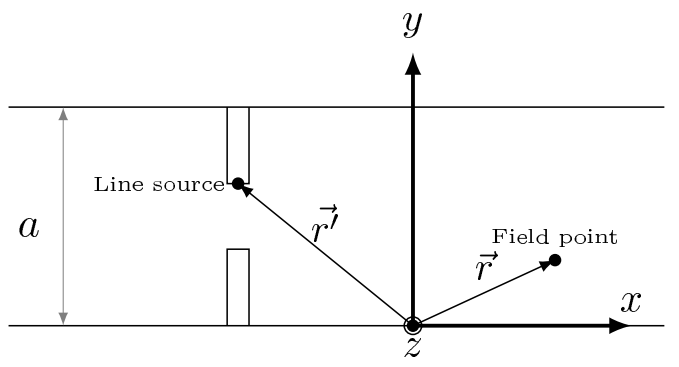

(b) Top view

Fig. 1. Rectangular waveguide with line source.

In this case we have to choose another criterion to make groups. Since our field spectrum is a set of guided modes $T E_{p 0}$ propagating either to the right $(+\hat{x})$ or to the left $(-\hat{x})$, we have to make groups according to their separation along the propagation axis $\hat{x}$, so we apply a $1-\mathrm{D}$ proximity criterion, and group the $N$ MoM cells (line sources) into $G$ groups as shown in the example of Fig. 2. The groups are always ordered from left to right.

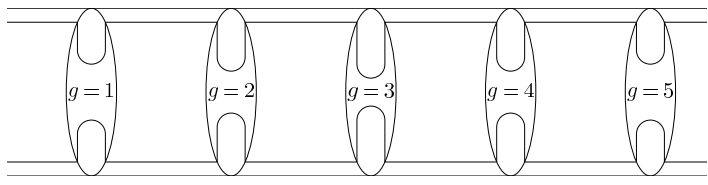

Fig. 2. Example of the 1-D grouping strategy for a 4-pole coupled cavities H-plane filter with rounded corners in the coupling windows. The MoM cells are grouped into $G=5$ groups.

As in the fast multipole method, we should now find a way to combine the field scattered by all the cells of the same group. Since the field scattered by a line source inside the parallel plates is expanded as a summation of modes propagating either to the right or to the left, we must aggregate the total field scattered by all the cells of a group to the right (propagating towards $+\hat{x}$ ), and to the left (propagating towards $-\hat{x})$. We must specify two reference planes, one for the set of modes propagating to the right, and another for the modes propagating to the left (see Fig. 3). 


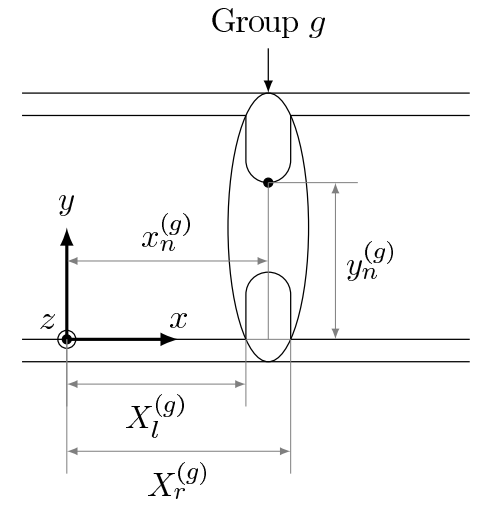

Fig. 3. Position of the reference planes for right and left propagating modes for the $g$-th group in an H-plane coupled cavities filter. The coordinates of the $n$-th MoM cell are also shown.

Let $g$ be the group that is being processed, $X_{l}^{(g)}$ the position in the $\hat{x}$ axis of the reference plane for left propagating modes, and $X_{r}^{(g)}$ for the right propagating modes. Let $N_{g}$ be the number of cells in the group $g$, and $j_{n}^{(g)}$ the weight of the MoM basis function of the $n$-th cell belonging to that group. Taking everything into consideration and applying the results of [18], we can compute the left $\left(\boldsymbol{e}_{l}\right)$ and right $\left(\boldsymbol{e}_{r}\right)$ propagating spectra as:

$$
\begin{aligned}
& e_{l, p}^{(g)}=\sum_{n=1}^{N_{g}} j_{n}^{(g)} A_{p n}^{-} e^{\jmath \beta_{x, p}\left(X_{l}^{(g)}-x_{n}^{(g)}\right)} \\
& e_{r, p}^{(g)}=\sum_{n=1}^{N_{g}} j_{n}^{(g)} A_{p n}^{+} e^{-\jmath \beta_{x, p}\left(X_{r}^{(g)}-x_{n}^{(g)}\right)}
\end{aligned}
$$

where $n$ identifies the MoM cell and $p \in[1 \ldots P]$ identifies the $T E_{p 0}$ guided mode, $T E_{P 0}$ being the higher mode considered for the analysis. The values of $A_{p n}^{+}$and $A_{p n}^{-}$can be found in [18].

To simplify the expressions we can put them in matrix form. For that purpose we define two new matrices of $P \times N_{g}$ elements:

$$
\begin{aligned}
& \beta_{l, p n}^{(g)}=A_{p n}^{-} e^{j \beta_{x, p}\left(x_{n}^{(g)}-X_{l}^{(g)}\right)} \\
& \beta_{r, p n}^{(g)}=A_{p n}^{+} e^{-j \beta_{x, p}\left(X_{r}^{(g)}-x_{n}^{(g)}\right)}
\end{aligned}
$$

Now the left and right propagating spectra of group $g$ can be computed in matrix form as:

$$
\begin{aligned}
& \boldsymbol{e}_{l}^{(g)}=\boldsymbol{\beta}_{l}^{(g)} \boldsymbol{j}^{(g)} \\
& \boldsymbol{e}_{r}^{(g)}=\boldsymbol{\beta}_{r}^{(g)} \boldsymbol{j}^{(g)}
\end{aligned}
$$

where $\boldsymbol{j}^{(g)}$ is a vector of size $N_{g} \times 1$ and stores the current weights of the MoM cells of the $g$-th group $\left(j_{n}^{(g)}, n \in\right.$ $\left.\left[1, \ldots, N_{g}\right]\right)$.

\section{B. Translation}

In the aggregation step we have combined the field scattered by all the cells of a group into two spectra, one propagating to the left and another one to the right. The spectra of each group are related to their own reference plane, so to compute the total field incident to a particular group $g$ we must translate all the incoming spectra from other groups to the same reference planes; one for the spectra that come from the left $X_{l}^{(g)}$ and another for the spectra that come from the right $X_{r}^{(g)}$.

The translation of a guided modes spectrum from one to another reference plane only requires a phase shift for each mode, and that depends on the distance from one to another reference plane. To translate the right propagating spectrum of group $g_{1}\left(\boldsymbol{e}_{r}^{\left(g_{1}\right)}\right)$, whose reference plane is $X_{r}^{\left(g_{1}\right)}$, to the left reference plane of group $g_{2}$ ( $g_{2}$ must be to the right of $\left.g_{1}\right)$, that is, to $X_{l}^{\left(g_{2}\right)}$, we just multiply $\boldsymbol{e}_{r}^{\left(g_{1}\right)}$ by a diagonal matrix $\alpha_{r}^{\left(g_{1}, g_{2}\right)}$ whose elements are

$$
\alpha_{r, p q}^{\left(g_{1}, g_{2}\right)}= \begin{cases}e^{-\jmath \beta_{x, p}\left(X_{l}^{\left(g_{2}\right)}-X_{r}^{\left(g_{1}\right)}\right)} & p=q \\ 0 & p \neq q\end{cases}
$$

Similarly, if we want to translate the left propagating spectrum of group $g_{2}$ to the right reference plane of group $g_{1}$, we must use the diagonal matrix $\alpha_{l}^{\left(g_{2}, g_{1}\right)}$, whose elements are

$$
\alpha_{l, p q}^{\left(g_{2}, g_{1}\right)}= \begin{cases}e^{j \beta_{x, p}\left(X_{r}^{\left(g_{1}\right)}-X_{l}^{\left(g_{2}\right)}\right)} & p=q \\ 0 & p \neq q\end{cases}
$$

Equations (8) and (9) are identical. Therefore

$$
\boldsymbol{\alpha}_{r}^{\left(g_{1}, g_{2}\right)}=\boldsymbol{\alpha}_{l}^{\left(g_{2}, g_{1}\right)}
$$

and therefore we only need to compute left or right translation matrices.

Once we have computed the translation matrices, we can write an expression for the total field incident to a particular group $g$ coming from the MoM cells of the rest of the groups:

$$
\begin{aligned}
\boldsymbol{i}_{l}^{(g)} & =\sum_{g^{\prime}=g+1}^{G} \boldsymbol{\alpha}_{l}^{\left(g^{\prime}, g\right)} \boldsymbol{e}_{l}^{\left(g^{\prime}\right)} \\
\boldsymbol{i}_{r}^{(g)} & =\sum_{g^{\prime}=1}^{g-1} \boldsymbol{\alpha}_{r}^{\left(g^{\prime}, g\right)} \boldsymbol{e}_{r}^{\left(g^{\prime}\right)}
\end{aligned}
$$

where $\boldsymbol{i}_{l}^{(g)}$ and $\boldsymbol{i}_{r}^{(g)}$ are, respectively, the incoming field spectra from the right and left. The groups are ordered from left to right, so that for a particular group $g$ the groups $g^{\prime}=[1, \ldots, g-1]$ are at the left side of group $g$, and the groups $g^{\prime}=[g+1, \ldots, G]$ are placed at the right side of group $g$, and $G$ being the total number of groups.

\section{Disaggregation}

Once we have computed for each group $g$ the incoming spectra from the right $\left(i_{l}^{(g)}\right)$ and from the left $\left(i_{r}^{(g)}\right)$, we must translate each spectrum and evaluate the field at the center $\left(x_{n}^{(g)}, y_{n}^{(g)}\right)$ of each MoM cell $n$ of group $g$. This step is called disaggregation.

The right and left incoming spectra that propagate, respectively, to the left $\left(\boldsymbol{i}_{l}^{(g)}\right)$ and to the right $\left(\boldsymbol{i}_{r}^{(g)}\right)$ can be translated and evaluated at the center $\left(x_{n}^{(g)}, y_{n}^{(g)}\right)$ of the $n$-th cell of group $g$ in matrix form using:

$$
\begin{aligned}
& \boldsymbol{b}_{l}^{(g)}=\boldsymbol{\gamma}_{l}^{(g)} \boldsymbol{i}_{l}^{(g)} \\
& \boldsymbol{b}_{r}^{(g)}=\boldsymbol{\gamma}_{r}^{(g)} \boldsymbol{i}_{r}^{(g)}
\end{aligned}
$$


where $\gamma_{l}^{(g)}$ and $\gamma_{r}^{(g)}$ are matrices of $N_{g} \times P$ elements of the form

$$
\begin{aligned}
& \gamma_{l, n p}^{(g)}=e^{\jmath \beta_{x, p}\left(x_{n}^{(g)}-X_{r}^{(g)}\right)} \sin \left(\frac{p \pi}{a} y_{n}^{(g)}\right) \\
& \gamma_{r, n p}^{(g)}=e^{-\jmath \beta_{x, p}\left(x_{n}^{(g)}-X_{l}^{(g)}\right)} \sin \left(\frac{p \pi}{a} y_{n}^{(g)}\right)
\end{aligned}
$$

The total field over each cell in group $g\left(\boldsymbol{b}_{\text {others }}^{(g)}\right)$ due to the scattering of the rest of the groups is

$$
\boldsymbol{b}_{\text {others }}^{(g)}=\boldsymbol{b}_{l}^{(g)}+\boldsymbol{b}_{r}^{(g)}
$$

$\boldsymbol{b}_{\text {others }}^{(g)}$ is a vector of $N_{g}$ elements. Each element represents the field scattered over the $n$-th cell in group $g$ by all the cells except those in group $g$.

Substituting (13) and (14) in (17)

$$
\boldsymbol{b}_{\text {others }}^{(g)}=\boldsymbol{\gamma}_{l}^{(g)} \boldsymbol{i}_{l}^{(g)}+\boldsymbol{\gamma}_{r}^{(g)} \boldsymbol{i}_{r}^{(g)}
$$

Substituting (11) and (12) in the former equation:

$$
\begin{aligned}
\boldsymbol{b}_{\text {others }}^{(g)} & =\boldsymbol{\gamma}_{l}^{(g)} \sum_{g^{\prime}=g+1}^{G} \boldsymbol{\alpha}_{l}^{\left(g^{\prime}, g\right)} \boldsymbol{e}_{l}^{\left(g^{\prime}\right)} \\
& +\gamma_{r}^{(g)} \sum_{g^{\prime}=1}^{g-1} \boldsymbol{\alpha}_{r}^{\left(g^{\prime}, g\right)} \boldsymbol{e}_{r}^{\left(g^{\prime}\right)}
\end{aligned}
$$

And finally substituting (6) and (7) we obtain:

$$
\begin{aligned}
\boldsymbol{b}_{\text {others }}^{(g)} & =\gamma_{l}^{(g)} \sum_{g^{\prime}=g+1}^{G} \boldsymbol{\alpha}_{l}^{\left(g^{\prime}, g\right)} \boldsymbol{\beta}_{l}^{\left(g^{\prime}\right)} \boldsymbol{j}^{\left(g^{\prime}\right)} \\
& +\gamma_{r}^{(g)} \sum_{g^{\prime}=1}^{g-1} \boldsymbol{\alpha}_{r}^{\left(g^{\prime}, g\right)} \boldsymbol{\beta}_{r}^{\left(g^{\prime}\right)} \boldsymbol{j}^{\left(g^{\prime}\right)}
\end{aligned}
$$

\section{Intra-group scattering}

Equation (20) provides the field scattered over the cells of group $g$ by all the cells of the other groups. To compute the total field incident for these cells, we must still consider the field that comes from the other cells of the same group $\left(\boldsymbol{b}_{\text {self }}^{(g)}\right)$.

$$
\boldsymbol{b}_{\text {self }}^{(g)}=\boldsymbol{Z}^{(g)} \boldsymbol{j}^{(g)}
$$

where $\boldsymbol{Z}^{(g)}$ is the submatrix of the whole MoM coefficients matrix of equation (1), that contains the interactions among cells of group $g$. In [18] there is a complete study of the value of the different parameters involved that have to be used to obtain a good trade-off between accuracy and efficiency.

Finally, the total field incident over the cells of group $g$ is

$$
\boldsymbol{b}^{(g)}=\boldsymbol{b}_{\text {self }}^{(g)}+\boldsymbol{b}_{\text {others }}^{(g)}
$$

where the whole vector $\boldsymbol{b}$, with the field incident to all the cells of the MoM problem, is

$$
\boldsymbol{b}=\left(\begin{array}{c}
\boldsymbol{b}^{(1)} \\
\boldsymbol{b}^{(2)} \\
\vdots \\
\boldsymbol{b}^{(G)}
\end{array}\right)=\boldsymbol{Z} \boldsymbol{j}
$$

With the new grouping strategy we avoid the computation of the whole MoM coefficients matrix $Z$, since we only compute the interactions among cells of the same group. Besides, we do not compute the matrix product $Z \boldsymbol{j}$. The computational cost is reduced and the only loss of accuracy comes from the truncation of the Green's function, as in [18]. The new grouping strategy does not add any additional loss of accuracy since we obtain exactly the same vector $\boldsymbol{Z} \boldsymbol{j}$ but using expression (22), instead of computing $\boldsymbol{Z}$ and multiplying by $j$.

\section{E. Number of modes}

The number of guided modes $T E_{p 0}$ considered for the aggregation, translation, and disaggregation stages cannot be infinite. The highest order used in all calculations is $P$ $(p \in[1,2, \cdots, P])$. The optimum value for $P$ depends on the distance between groups. If we call this distance $D$, the criterion that we have chosen is to ensure for each frequency point that the scattered field corresponding to the highest order mode $T E_{P 0}$ vanishes from one group to another below some threshold value $\eta$, so that

$$
e^{-\gamma_{P} D}=e^{-\sqrt{\frac{P \pi}{a}-k^{2}} D} \leq \eta
$$

And therefore $P$ must be

$$
P \geq \frac{a}{\pi}\left[\left(\frac{\ln \eta}{D}\right)^{2}+k^{2}\right]
$$

where $\eta=10^{-3}$ has been proved to provide a good tradeoff between accuracy and efficiency.

\section{F. Computational cost}

Below we are going to analyze which is the number of operations required for the computation of the matrix product $\boldsymbol{b}=\boldsymbol{Z} \boldsymbol{j}$ using the new grouping strategy, that is, equation (22). The number of operations needed for filling all the matrices $\left(\boldsymbol{Z}^{(g)}, \boldsymbol{\beta}_{r}^{(g)}, \boldsymbol{\beta}_{l}^{(g)}, \boldsymbol{\alpha}_{r}^{(g)}, \boldsymbol{\alpha}_{l}^{(g)}, \boldsymbol{\gamma}_{r}^{(g)}, \boldsymbol{\gamma}_{l}^{(g)}\right.$ ) is not considered since this is done only once, while the matrix product $\boldsymbol{Z} \boldsymbol{j}$ is performed several times by the iterative technique (biconjugate gradients stabilized) that solves the MoM matrix system, so these matrix products are the most time consuming process. The number of operations needed to evaluate the matrix product $\boldsymbol{Z} \boldsymbol{j}$ for each step of the grouping strategy is analyzed separately:

1) Aggregation step: the products $\boldsymbol{\beta}_{l}^{(g)} \boldsymbol{j}^{(g)}$ and $\boldsymbol{\beta}_{r}^{(g)} \boldsymbol{j}^{(g)}$ must be computed for all the groups (there are $G$ groups). For all these products we need $2 G P N_{g}=2 P N$ operations since $N=G N_{g}$, and $N$ is the number of MoM cells.

2) Translation step: we need to compute the products $\boldsymbol{\alpha}_{r}^{\left(g^{\prime}, g\right)} \boldsymbol{e}_{r}^{\left(g^{\prime}\right)}$ for $g^{\prime}=1, \cdots, g-1$ and $\boldsymbol{\alpha}_{l}^{\left(g^{\prime}, g\right)} \boldsymbol{e}_{l}^{\left(g^{\prime}\right)}$ for $g^{\prime}=g+1, \cdots, G$. Since $\boldsymbol{\alpha}_{r}^{\left(g^{\prime}, g\right)}$ and $\boldsymbol{\alpha}_{l}^{\left(g^{\prime}, g\right)}$ are diagonal matrices of size $P$, those products suppose $(G-1) P$ operations. And this must be repeated for all the groups with $g=1,2, \ldots G$. In the end, we need $G(G-1) P$ operations for this step.

3) Disaggregation step: the products $\gamma_{l}^{(g)} i_{r}^{(g)}$ and $\gamma_{r}^{(g)} \boldsymbol{i}_{l}^{(g)}$ must be computed for all groups. That means $2 G N_{g} P=$ $2 P N$ operations for this step. 
4) The computational cost of computing the field scattered by cells of the same group over themselves $\left(\boldsymbol{Z}^{(g)} \boldsymbol{j}^{(g)}\right)$ supposes $G N_{g}^{2}=N^{2} / G$ operations.

Finally, the total number of operations needed with the new grouping strategy to compute the vector $\boldsymbol{b}=\boldsymbol{Z} \boldsymbol{j}$ is of the order of:

$$
C_{T O T}=O\left(N^{2} / G\right)+O(4 P N)+O(G(G-1) P)
$$

If the number of MoM cells $N$ is much bigger than the number of groups $G$, and groups are placed far away so that $P$ is small compared with $N$, we expect a number of operations of the order of $O\left(N^{2} / G\right)$.

If we do not use the grouping strategy, and compute the whole matrix $\boldsymbol{Z}$, and then multiply by vector $\boldsymbol{j}$, this matrix product needs a number of operations of the order of

$$
C_{T O T}=O\left(N^{2}\right)
$$

The order in number of operations can therefore be reduced up to a factor of $G$ provided that $N$ is much bigger than $G$ and $P$. However the relation between $N, G$ and $P$ depends on the particular geometry that we are analyzing, since we can not freely chose the number of subgroups $G$ as in FMM, and $P$ depends on the separation along the propagation axis of the different groups. The same reduction of the computational time applies to the computer memory needed to store the matrices involved in the calculations when the grouping strategy is used.

This result is especially important since the MoM matrix system of (1) is solved using an iterative technique that does not directly solve the matrix system of (1), but instead approximates the solution iteratively through multiple matrixvector products of the form $Z \boldsymbol{a}$. With the new grouping strategy the cost of these products and the memory needed is reduced by $G$, the number of groups used in the grouping strategy.

Besides, with the new grouping strategy it is not necessary to compute the whole matrix $\boldsymbol{Z}$, since only the smaller matrices $\boldsymbol{Z}^{(g)}$ with $g \in[1, \cdots, G]$ must be computed.

\section{RESULTS}

The accuracy and efficiency of the new grouping strategy is tested with the analysis of five $\mathrm{H}$-plane filters in rectangular waveguide. These filters, whose top view is shown in Fig. 4, are:

1) Four cavity H-plane coupled cavity filters. The coupling windows present rounded corners (radii $r=1 \mathrm{~mm}$ ) due to low cost manufacturing techniques [47].

2) Six cavity H-plane coupled cavity filters. The coupling windows present rounded corners (radii $r=2 \mathrm{~mm}$ ) due to low cost manufacturing techniques [48].

3) Six cavity H-plane coupled cavity filters. The resonant cavities present rounded corners (radii $r=3 \mathrm{~mm}$ ) due to low cost manufacturing techniques [48].

4) Bandpass filter with seven round rods [49].

5) Nine pole bandpass filter with wedges [50].

The frequency response (reflection coefficient) of these five filters has been computed using the MoM method of [18] accelerated with the new grouping strategy presented here.
The biconjugate gradients stabilized method has been used to accelerate the solution of the MoM matrix system of (1). Since the MoM method uses the Green's function of a line source between two parallel plates, only the portions of the metallic surface of the structure that are outside of the parallel plate waveguide must be segmented using MoM. This means that only the coupling windows for the first three filters, or the round rods and wedges for the last two filters, must be segmented into MoM cells. The new grouping strategy groups the MoM cells into $G$ groups, so that those MoM cells that are close together in the propagation direction must be enclosed in the same group for the grouping strategy to be efficient. This means, for instance, that the first filter is analyzed with all MoM cells split into $G=5$ groups, one for each coupling window, and similarly the MoM cells of the fourth filter are grouped into $G=7$ groups, one for each round rod.

The results of the analysis of the five filters are presented at Fig. 5, where they are compared with other analysis methods [47]-[50]. Fig. 5 shows a very good agreement with the results from the literature for the five filters analyzed.

Once the accuracy of the new method is fully proven, we will test the efficiency improvement of the new grouping strategy. For that purpose we present in Table I the CPU time (in seconds per frequency point) required to analyze each one of the five H-plane filters of Fig. 4. Table I shows both the CPU time required by the MoM with the Green's function of a line source between parallel plates [18] and the CPU time of the same analysis method accelerated with the new grouping strategy.

\begin{tabular}{|l|c|c|c|}
\hline Filter & MoM [18] & MoM+Grouping & Reduction \\
\hline \hline$r=1 \mathrm{~mm}$ windows & $0.91 \mathrm{~s} / \mathrm{pt}$ & $0.31 \mathrm{~s} / \mathrm{pt}$ & $66 \%$ \\
\hline$r=2 \mathrm{~mm}$ windows & $5.18 \mathrm{~s} / \mathrm{pt}$ & $1.91 \mathrm{~s} / \mathrm{pt}$ & $63 \%$ \\
\hline$r=3 \mathrm{~mm}$ cavities & $8.69 \mathrm{~s} / \mathrm{pt}$ & $3.03 \mathrm{~s} / \mathrm{pt}$ & $65 \%$ \\
\hline Round rods & $0.57 \mathrm{~s} / \mathrm{pt}$ & $0.34 \mathrm{~s} / \mathrm{pt}$ & $40 \%$ \\
\hline Wedges & $0.59 \mathrm{~s} / \mathrm{pt}$ & $0.29 \mathrm{~s} / \mathrm{pt}$ & $51 \%$ \\
\hline
\end{tabular}

TABLE I

COMPUTATIONAL COST FOR THE ANALYSIS OF THE FIVE FILTERS OF Fig. 4. COMPARISON BETWEEN THE MOM METHOD OF [18] AND THE SAME METHOD ACCELERATED WITH THE NEW GROUPING STRATEGY PROPOSED IN THIS PAPER. CPU TIME IS IN SECONDS PER FREQUENCY POINT.

Results from Table I show that the time is reduced by a significant factor of 2 or 3, depending on the filter. However, the number of groups $G$ varies from 5 in the first filter to 10 in the last filter. As mentioned before, a time reduction of $G$ could be expected when the number of MoM cells is much bigger than the number of groups $G$ and the number of guided modes $P$. Since $G$ and $P$ are not selectable, we can not ensure a time reduction of $G$. In the geometry of the last two filters there is a much shorter metallic surface for each group, so the ratio $N / G=N_{g}$, that is, the number of MoM cells per group, is much smaller, and so the last term in equation (26) is more important than in the case of the three first filters. This results in a smaller time reduction. However, this time reduction becomes especially significant in design processes where many simulations of the structure must be performed in order to obtain the optimal values of the design parameters. 


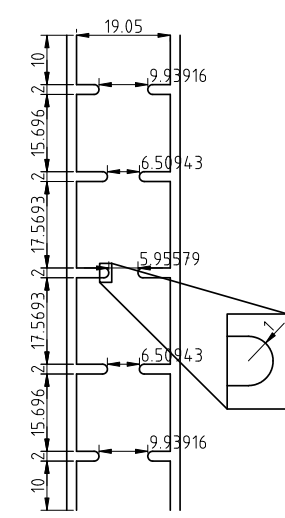

(a)

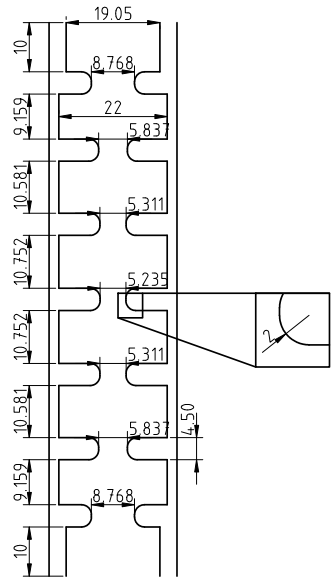

(b)

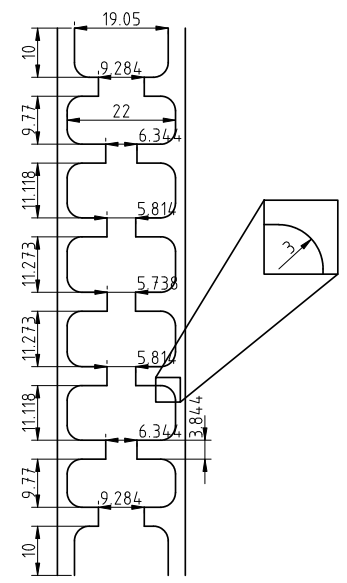

(c)

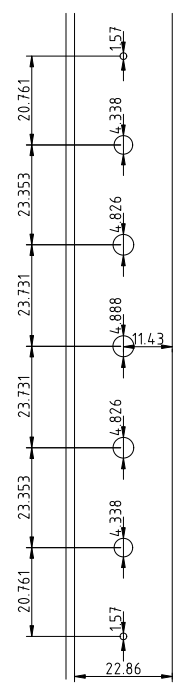

(d)

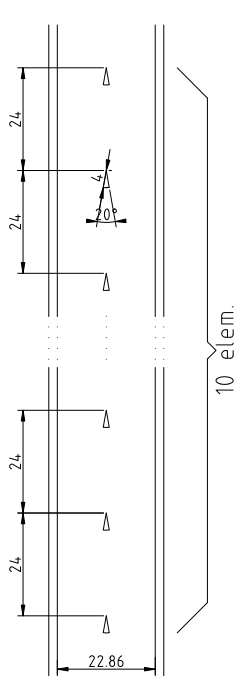

(e)

Fig. 4. H-plane filters (measures in mm.). a) Four cavity filter with rounded corners at coupling windows $r=1$ mm, b) Six cavity filter with rounded corners at coupling windows $r=2 \mathrm{~mm}$, c) Six cavity filter with rounded corners at resonant cavities $r=3 \mathrm{~mm}$. d) Round rod bandpass filter from [49]. e) Wedge bandpass filter from [50].

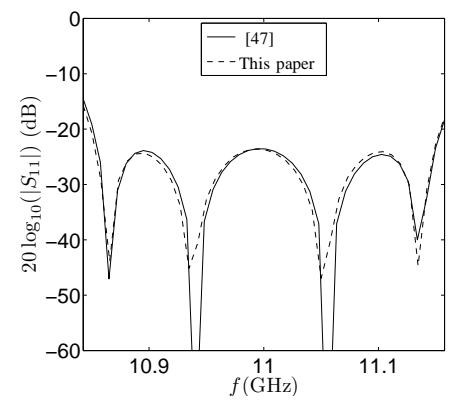

(a)

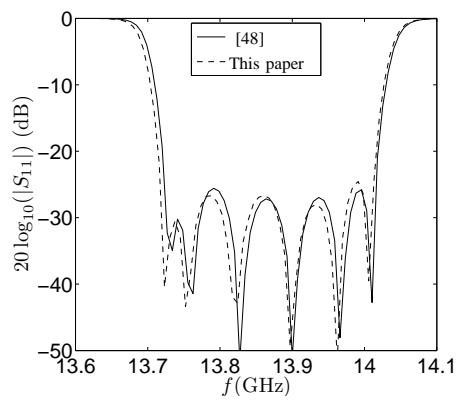

(b)

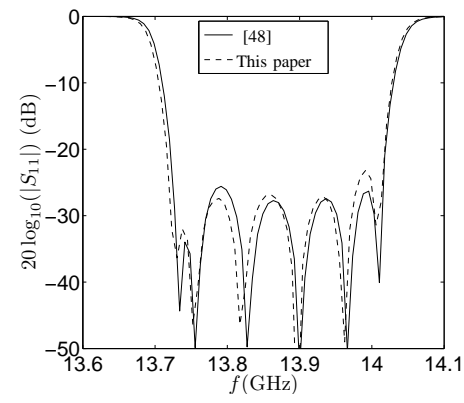

(c)

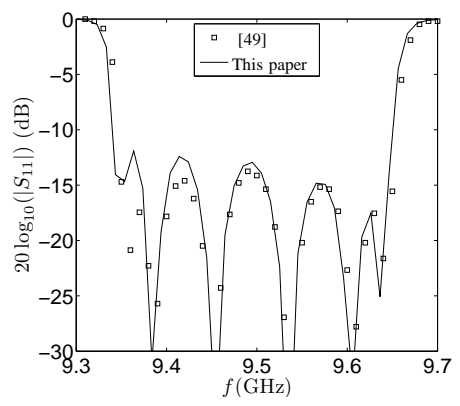

(d)

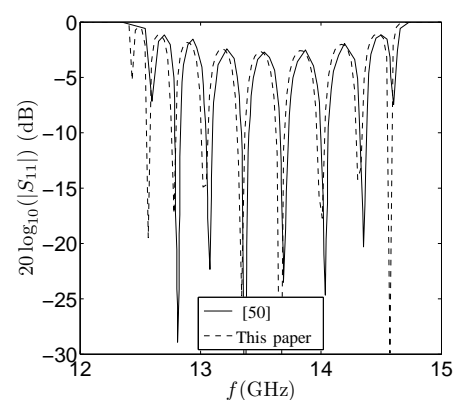

(e)

Fig. 5. Frequency response of the H-plane filters of Fig. 4 compared with results from the bibliography.

There are other factors that could explain that the time reduction is lower than $G$, such as the fact that we have not considered other processes that consume time, such as the computation of the elements needed to fill the matrices $Z^{(g)}$, $\boldsymbol{\beta}_{r}^{(g)}, \boldsymbol{\beta}_{l}^{(g)}, \boldsymbol{\alpha}_{r}^{(g)}, \boldsymbol{\alpha}_{l}^{(g)}, \boldsymbol{\gamma}_{r}^{(g)}$ and $\boldsymbol{\gamma}_{l}^{(g)}$, or the time spent in reading and writing in memory and disk.

A similar time reduction could have been obtained with a classical segmentation approach [51], where a generalized matrix of circuit parameters is obtained for each group and then all the matrices are cascaded in order to obtain the global circuit parameters of the problem. However, the segmentation strategy presented here provides much more accurately the field inside the structure, since all the currents in all the structure are obtained at the same time, and the field is directly computed from the amplitudes of these currents. With a classical segmentation structure a complex process must be followed in order to find the modal amplitudes in each intermmediate point of the structure. Next these modal amplitudes are used to expand the field inside each segment of the filter, and small discontinuities usually appear between adjacent segments due to loss of accuracy inherent to the segmentation process. 


\section{CONClusions}

A new grouping strategy has been presented in this paper. This new strategy accelerates the MoM method with the Green's function of a line source between two parallel plates presented in [18]. The efficiency improvement of the new grouping strategy is especially important when the MoM matrix system is solved using an iterative technique such as conjugate gradients, biconjugate gradients, biconjugate gradients stabilized, and others; since these techniques require multiple matrix-vector products of the MoM coefficients matrix by a vector that iteratively converges to the solution. This matrix product is not solved directly with the new grouping strategy, but is instead solved without loss of accuracy using a more efficient three-step procedure.

The accuracy and efficiency of the new grouping strategy has been tested with the analysis of five H-plane filters. The reflection coefficient of these filters has been successfully compared with results from the technical literature, and a significant time reduction of $40 \%, 50 \%$ or $60 \%$ has been obtained depending on the type of filter and the number of MoM cells per group.

\section{ACKNOWLEDGEMENTS}

We would like to thank the R\&D\&I Linguistic Assistance Office, Universidad Politécnica de Valencia (Spain), for granting financial support for the linguistic revision of this paper.

\section{REFERENCES}

[1] R. F. Harrington, Field Computation by Moment Methods, ser. IEEE Press / OUP Series on Electromagnetic Wave Theory. IEEE Press / OUP, 1993.

[2] A. F. Peterson, S. L. Ray, and R. Mittra, Computational Methods for Electromagnetics, ser. IEEE Press / OUP Series on Electromagnetic Wave Theory. IEEE Press / OUP, 1997.

[3] W. C. Chew, J.-M. Jin, E. Michielssen, and J. Song, Fast and Efficient Algorithms in Computational Electromagnetics. Norwood, Massachusetts: Artech House, 2001.

[4] M. Guglielmi and C. Newport, "Rigorous, multimode equivalent network representation of inductive discontinuities," IEEE Transactions on Microwave Theory and Techniques, vol. 38, no. 11, pp. 1651-1659, November 1990.

[5] T. Rozzi, F. Moglie, A. Morini, W. Gulloch, and M. Politi, "Accurate full-band equivalent circuits of inductive posts in rectangular waveguide," IEEE Transactions on Microwave Theory and Techniques, vol. 40, no. 5, pp. 1000-1009, May 1992.

[6] G. Conciauro, P. Arcioni, M. Bressan, and L. Perregrini, "Wideband modeling of arbitrarily shaped H-plane waveguide components by the BI-RME method,' IEEE Transactions on Microwave Theory and Techniques, vol. 44, no. 7, pp. 1057-1066, July 1996.

[7] M. Guglielmi, "Simple CAD procedure for microwave filters and multiplexers," IEEE Transactions on Microwave Theory and Techniques, vol. 42, no. 7, pp. 1347-1352, July 1994.

[8] F. Arndt, R. Beyer, J. M. Reiter, T. Sieverding, and T. Wolf, "Automated design of waveguide components using hybrid modematchning/numerical EM building-blocks in optimization-oriented CAD frameworks-State-of-the-art and recent advances," IEEE Transactions on Microwave Theory and Techniques, vol. 45, no. 5, pp. 747-760, May 1997.

[9] S. Yin, T. Vasilyeva, and P. Pramanick, "Use of three-dimensional field simulators in the synthesis of waveguide round rod bandpass filters," International Journal of RF and Microwave Computer-Aided Engineering, vol. 8, no. 6, pp. 484-497, December 1998.

[10] R. H. Macphie and K.-L. Wu, "A full wave modal analysis of arbitrarily shaped waveguide discontinuities using the finite plane-wave series expansion," IEEE Transactions on Microwave Theory and Techniques, vol. 47, no. 2, pp. 232-237, February 1999.
[11] H. Esteban, S. Cogollos, V. E. Boria, A. A. San Blas, and M. Ferrando, "A new hybrid mode-matching/numerical method for the analysis of arbitrarily shaped inductive obstacles and discontinuities in rectangular waveguides," IEEE Transactions on Microwave Theory and Techniques, vol. 50, no. 4, pp. 1219-1224, April 2002.

[12] C.-T. Tai, Dyadic Green Functions in Electromagnetic Theory. Piscataway, New Jersey: IEEE Press, 1993.

[13] R. E. Collin, Field Theory of Guided Waves, 2nd ed. New York: IEEE Press, 1991.

[14] Y. Leviatan, P. G. Li, A. T. Adams, and J. Perini, "Single post inductive obstacle in rectangular waveguide," IEEE Transactions on Microwave Theory and Techniques, vol. 31, no. 10, pp. 806-812, October 1983.

[15] Y. Leviatan, D.-H. Shau, and A. T. Adams, "Numerical study of the current distribution on a post in a rectangular waveguide," IEEE Transactions on Microwave Theory and Techniques, vol. 32, no. 10, pp. 1411-1415, October 1984.

[16] P. G. Li, A. T. Adams, Y. Leviatan, and J. Perini, "Multiple post inductive obstacles in rectangular waveguide," IEEE Transactions on Microwave Theory and Techniques, vol. 32, no. 4, pp. 365-373, April 1984.

[17] H. Auda and R. Harrington, "Inductive posts and diaphragms of arbitrary shape and number in a rectangular waveguide," IEEE Transactions on Microwave Theory and Techniques, vol. 32, no. 6, pp. 606-613, June 1984.

[18] A. Belenguer, H. Esteban, V. E. Boria, J. V. Morro, and C. Bachiller, "Efficient analysis of two-port $\mathrm{H}$ plane devices using the green's function of a line source inside parallel plates," IET Microwaves, Antennas and Propagation, Accepted, to be published in 2008.

[19] V. Rokhlin, "Rapid solution of integral equations of scattering theory in two dimensions," Journal of Computational Physics, vol. 86, no. 2, pp. 414-439, February 1990.

[20] N. Engheta, W. D. Murphy, V. Rokhlin, and M. Vassiliou, "The Fast Multipole Method (FMM) for electromagnetic scattering problems," IEEE Trans. Antennas Propagat., vol. 40, no. 6, pp. 634-641, June 1992.

[21] R. Coifman, V. Rokhlin, and S. Wandzura, "The Fast Multipole Method for the wave equation: A pedestrian prescription," IEEE Antennas Propagat. Magazine, vol. 35, no. 3, pp. 7-12, February 1993.

[22] J. Song and W. C. Chew, "Fast Multipole Method solution of combined field integral equation," in 11th Annual Review of Progress in Applied Computational Electromagnetics, vol. 1. Monterey, California: Applied Computational Electromagnetics Society, March 1995, pp. 629-636.

[23] J. Song, C.-C. Lu, and W. C. Chew, "Multilevel Fast Multipole Algorithm for electromagnetic scattering by large complex objects," IEEE Trans. Antennas Propagat., vol. 45, no. 10, pp. 1488-1493, October 1997.

[24] J. Song and W. C. Chew, "Multilevel Fast Multipole Algorithm for solving combined field integral equation of electromagnetic scattering," Microwave and Optical Technology Letters, vol. 10, no. 1, pp. 14-19, September 1995.

[25] X. Q. Sheng, J.-M. Jin, J. Song, W. C. Chew, and C.-C. Lu, "Solution of combined-field integral equation using Multilevel Fast Multipole Algorithm for scattering by homogeneous bodies," IEEE Trans. Antennas Propagat., vol. 46, no. 11, pp. 1718-1726, November 1998.

[26] R. Barrett, M. Berry, T. F. Chan, J. Demmel, J. Donato, J. Dongarra, V. Eijkhout, R. Pozo, C. Romine, and H. V. der Vorst, Templates for the Solution of Linear Systems: Building Blocks for Iterative Methods, 2nd ed. Philadelphia, PA: SIAM, 1994.

[27] Y. Saad, Iterative Methods for Sparse Linear Systems, 2nd ed. SIAM, January 2003.

[28] J. Lee, J. Zhang, and C.-C. Lu, "Performance of preconditioned Krylov iterative methods for solving hybrid integral equations in electromagnetics," Journal of Applied Computational Electromagnetics Society, vol. 18 , no. 3, pp. 54-61, 2003.

[29] A. F. Peterson and R. Mittra, "Convergence of the Conjugate Gradients Method when applied to matrix equations representing electromagnetic scattering problems," IEEE Trans. Antennas Propagat., vol. 34, no. 12, pp. 1447-1454, December 1986.

[30] J. Sarvas, "Performing interpolation and anterpolation entirely by Fast Fourier Transform in the 3-D Multilevel Fast Multipole Algorithm,' SIAM Journal on Numerical Analysis, vol. 41, no. 6, pp. 2180-2196, 2003.

[31] O. Ergül and L. Gürel, "Enhancing the accuracy of the interpolations and anterpolations in MLFMA," IEEE Antennas and Wireless Propagation Letters, vol. 5, no. 1, pp. 467-470, December 2006.

[32] Özgür Ergül and L. Gürel, "Linear-linear basis functions for MLFMA solutions of Magnetic-Field and Combined-Field Integral Equations," 
IEEE Trans. Antennas Propagat., vol. 55, no. 4, pp. 1103-1110, April 2007.

[33] W. B. Lu, T. J. Cui, and Z. Hui, "Acceleration of Fast Multipole Method for large-scale periodic structures with finite sizes using sub-entiredomain basis functions," IEEE Trans. Antennas Propagat., vol. 55, no. 2 , pp. 414-421, February 2007.

[34] D. Z. Ding, P. L. Rui, R. S. Chen, and Z. H. Fan, "Loose GMRES method for efficient solution of EFIE in the MLFMA context," $M i$ crowave and Optical Technology Letters, vol. 49, no. 11, pp. 2661-2665, November 2007.

[35] P. L. Rui, R. S. Chen, D. X. Wang, and E. K.-N. Yung, "Spectral twostep preconditioning of multilevel fast multipole algorithm for the fast monostatic rcs calculation," IEEE Trans. Antennas Propagat., vol. 55, no. 8, pp. 2268-2275, August 2007.

[36] T. Maias and L. Guerel, "Incomplete LU preconditioning with the Multilevel Fast Multipole Algorithm for electromagnetic scattering," SIAM Journal on Scientific Computing, vol. 29, no. 4, pp. 1476-1494, 2007.

[37] X. M. Pan and X. Q. Sheng, "A highly efficient parallel approach of Multi-Level Fast Multipole Algorithm," Journal of Electromagnetic Waves and Applications, vol. 20, no. 8, pp. 1081-1092, June 2006.

[38] C. Waltz, S. Kubilay, M. A. Carr, B. C. Usner, and J. L. Volakis, "Massive parallel Fast Multipole Method solutions of large electromagnetic scattering problems," IEEE Trans. Antennas Propagat., vol. 55, no. 6, pp. 1810-1816, June 2007

[39] S. Velamparambil and W. C. Chew, "Analysis and performance of a distributed memory multilevel fast multipole algorithm," IEEE Trans. Antennas Propag., vol. 53, no. 8, pp. 2719-2727, 2005.

[40] O. Ergul and L. Gurel, "Hierarchical parallelisation strategy for multilevel fast multipole algorithm in computational electromagnetics," Electron. Lett., vol. 44, no. 1, pp. 3-5, 2008.

[41] _ , "Efficient parallelization of the multilevel fast multipole algorithm for the solution of large-scale scattering problems," IEEE Trans. Antennas Propagat., vol. 56, no. 8, pp. 2335-2345, 2008.

[42] Z. O. Zaw, E. P. Li, and L. W. Li, "Analysis and design on aperture antenna systems with large electrical size using multilevel fast multipole method," Journal of Electromagnetic Waves and Applications, vol. 19 no. 11, pp. 1485-1500, September 2005.

[43] L. Li and Y. Xie, "Efficient algorithm for analyzing microstrip antennas using fast-multipole algorithm combined with fixed real-image simulated method," Journal of Electromagnetic Waves and Applications, vol. 20, no. 15, pp. 2177-2188, December 2006.

[44] P. Wang and Y. Xie, "Scattering and radiation problem of surface/surface junction structure with Multilevel Fast Multipole Algorithm," Journal of Electromagnetic Waves and Applications, vol. 20, no. 15, pp. 2189-2200, December 2006.

[45] X. Liu, C. Liang, and X. Zhao, "Analysis of waveguide slot antennas using MLFMA," Microwave and Optical Technology Letters, vol. 50, no. 1, pp. 65-68, January 2008.

[46] X. W. Zhao, C. H. Liang, and L. Liang, "Multilevel Fast Multipole Algorithm for radiation characteristics of shipborne antennas above seawater," Progress In Electromagnetics Research, vol. PIER 81, pp. 303-314, 2008

[47] J. V. Morro, H. Esteban, V. E. Boria, C. Bachiller, S. Cogollos, and A. Coves, "Efficient automated design of H-plane filters with rounded corners using ASM with a segmentation strategy and hybrid optimization techniques," in 2004 IEEE AP-S Int. Symp. Dig., vol. 3, Monterey, California, June 2004, pp. 2735-2738.

[48] J. V. Morro, H. Esteban, V. E. Boria, C. Bachiller, and A. Belenguer, "Optimization techniques for the efficient design of low-cost satellite filters considering new light materials," International Journal of $R F$ and Microwave Computer-Aided Engineering, vol. DOI: 10.1002/mmce.20264, pp. 1-8, February 2008.

[49] S. Yin, T. Vasilyeva, and P. Pramanick, "Use of three-dimensional field simulators in the synthesis of waveguide round rod bandpass filters," International Journal of $R F$ and Microwave Computer-Aided Engineering, vol. 8, no. 6, pp. 484-497, 1998.

[50] R. Lech and J. Mazur, "Propagation in rectangular waveguides periodically loaded with cylindrical posts," IEEE Microwave and Wireless Components Letters, vol. 14, no. 4, pp. 177-179, April 2004.

[51] C. Bachiller, H. Esteban, V. E. Boria, A. Belenguer, and J. V. Morro, "Efficient technique for the cascade connection of multiple two port scattering matrices," IEEE Transactions on Microwave Theory and Techniques, vol. 55, no. 9, pp. 1880-1886, September 2007.

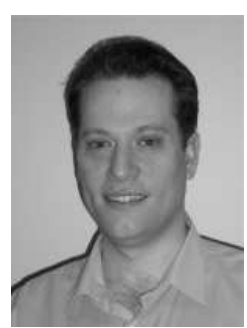

Ángel Belenguer Martínez received a degree in telecommunications engineering from the Universidad Politécnica de Valencia (UPV), Spain, in 2000. He joined the Universidad de Castilla-La Mancha in 2000. His research interests include methods for the full- wave analysis of open-space and guided multiple scattering problems, and the acceleration of electromagnetic analysis methods using the wavelets, the fast multipole method, the multilevel fast multipole algorithm, and the use of specific basis and green functions for the method of moments.

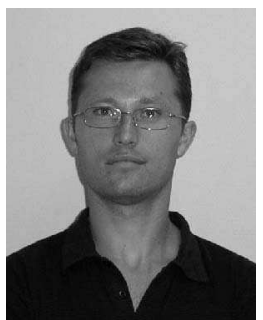

Héctor Esteban González received a degree in telecommunications engineering from the Universidad Politécnica de Valencia (UPV), Spain, in 1996, and a Ph.D. degree in 2002. He worked with the Joint Research Centre, European Commission, Ispra, Italy. In 1997, he was with the European Topic Centre on Soil (European Environment Agency). He rejoined the UPV in 1998. His research interests include methods for the full- wave analysis of open-space and guided multiple scattering problems, CAD design of microwave devices, electromagnetic characterization of dielectric and magnetic bodies, and the acceleration of electromagnetic analysis methods using the wavelets and the fast multipole method.

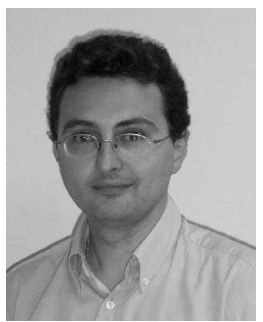

Vicente E. Boria Esbert received the Ingeniero de Telecomunicación and the Doctor Ingeniero de Telecomunicación degrees from the Universidad Politécnica de Valencia, Spain, in 1993 and 1997. In 1993 he joined the Universidad Politécnica de Valencia where he is Full Professor since 2003. In 1995 and 1996 he was held a Spanish Trainee position with the European Space research and Technology Centre (ESTEC)-European Space Agency (ESA). He has served on the Editorial Boards of the IEEE Transactions on Microwave Theory and Techniques. His current research interests include numerical methods for the analysis of waveguide and scattering structures, automated design of waveguide components, radiating systems, measurement techniques, and power effects in passive waveguide systems.

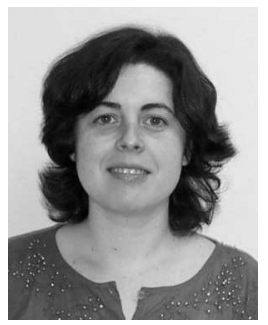

Carmen Bachiller received her degree in communication engineering from the Universidad Politécnica de Valencia in 1996. She worked from 1997 to 2001 in the ETRA I+D company as a project engineer in research and development on automatic traffic control, public transport management and public information systems using telecommunication technology. In 2001 she joined the Communication Department of the Universidad Politécnica de Valencia as an assistant lecturer. She is teaching signal and systems theory and microwaves. She has participated in several teaching innovation projects. She is now working in her Ph.D. thesis on electromagnetism and radiofrequency circuits. 


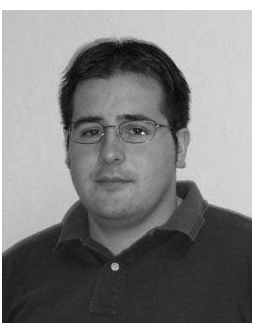

José Vicente Morro received the telecommunications engineering degree from the Universidad Politécnica de Valencia (UPV), Valencia, Spain, in 2001, and is currently pursuing his Ph.D. degree at the UPV. In 2001, he became a Research Fellow with the Departamento de Comunicaciones, UPV. In 2003 he joined the Signal Theory and Communications Division, Universidad Miguel Hernández, where he was a Lecturer. In 2005, he rejoined the Departamento de Comunicaciones, UPV, as an Assistant Lecturer. His current interests include CAD design of microwave devices and EM optimization methods. 